Classification

Physics Abstracts

$07.80-73.90$

\title{
Observation of Al-lines in LSI devices by ultra-high voltage electron microscope
}

\author{
Akio Takaoka, Noriyuki Okaya and Katsumi Ura \\ Department of Electronic Engineering, Faculty of Engineering, Osaka University, Yamada-oka 2-1, \\ Suita, Osaka, 565, Japan
}

(Received 12 March 1993; accepted 7 June 1993)

\begin{abstract}
To clarify the mechanism caused by multi-layer effect, the ultra-HVEM at $2 \mathrm{MeV}$ is powerful because it has an excellent penetration power for specimens. By thinning the Si-substrate of LSI devices, we can carry out in-situ observations of phenomena arising in the process layers; in particular, we can observe the specimens without removing the passivation layers that strongly affect the generation of these phenomena. We show how the orientation of Al-lines depends on the step of process and how it relates to the incidence of stressmigration voids. From in-situ observation of electromigration in Al-lines with bamboo structure, it is seen that the main route of electromigration is the interface between the line and the passivation layer, and the migration is enhanced when a small gap exits in the interface.
\end{abstract}

\section{Introduction.}

Although many multi-layer devices represented by large scale integrated (LSI) devices are produced in the industrial world, there are many phenomena whose mechanisms are not clear yet. The mechanisms in multi-layers are thought to be quite different from those in a single layer, because stress, diffusion, reaction, and potential gradient are generated between layers. So, the mechanisms induced by the multi-layer effect are complicated. As the line width of LSI circuits becomes less than one micron meter, this effect causes serious problems such as the reduction of reliability due to stressmigration or electromigration; the line is broken because of the migration of constituent atoms, which occurs due to the stress from the passivation layer or the collision of electron flow.

On the other hand, the ultra-High Voltage Electron Microscope (HVEM) in Osaka University has an excellent penetration power (Fujita et al., 1972) and observable thickness at $2 \mathrm{MeV}$ corresponds to the thickness of total process layers of LSI devices. When the Si-substrate is thinned down, we can observe the projection image of its device layers. The perpendicular observation is favorable since the process layers do not lose so much inherent characteristics in the preparation of TEM specimens. This fact means that it is suitable for the in-situ observation of LSI devices (Okabayashi et al., 1991, Takaoka et al., 1991). 
In this paper, we introduce the attractive points of the perpendicularly observation of LSI devices by ultra-HVEM, and then show some results concerning the observation of Al-lines which are produced by the same process as LSI devices.

\section{In-situ observatin of LSI devices.}

Let us summarize the effective and favorable points in the in-situ observation of the process layers of LSI devices with the ultra-HVEM.

Usual LSI devices are composed of multiple layers processed on a Si-substrate. The total thickness of these layers is about $2 \sim 5 \mu \mathrm{m}$. The top passivation layer is made by amorphous $\mathrm{SiO}_{2}$ and/or SiN whose thickness is about $1 \mu \mathrm{m}$. This amorphous layer blurs and dims the image of the fine structure of circuits; especially in the case of a 100 or $200 \mathrm{keV}$ class TEM, this effect is remarkable. So, in the perpendicular observation of LSI devices with such a TEM, the passivation layer is removed in most cases. The removal of passivation layers, however, changes the conditions of stress and surface state, and changes the mechanism itself.

The ultra-HVEM enables us to observe a specimen with thick amorphous layers. This is easily understood from the fact that as electron energy increases, the observed image becomes clearer and brighter at constant specimen thickness. This results from the following facts: 1) The transmissivity of electrons through a thick amorphous layer increases as the electron energy increases, and this is remarkable for a large aperture angle (Reimer et al., 1968). The effect of the quantum noise during in-situ observation is reduced. 2) The scattering angle becomes smaller for higher energy electron and this prevents the blur of image by the electron multiple scattering especially in a thick amorphous layer. 3) The ratio of energy loss to primary energy is small. This reduces the effect of chromatic aberration of lens systems.

Experimentally, it has been shown already (Fujita et al., 1972) that the maximum observable thickness at $2 \mathrm{MeV}$ is more than ten times larger than that of a $100 \mathrm{keV}$ TEM and is several $\mu \mathrm{m}$ for light atoms such as $\mathrm{Si}, \mathrm{O}, \mathrm{Al}$, etc. This thickness is comparable to that of processed layers. In the operating conditions of LSI devices, the main factors acting on the layers are temperature, stress and applied current. The atoms in the layer will migrate due to these factors and this may worsen the characteristics of LSI elements, for instance, causing the disconnection of a line in LSI devices. Therefore, it is important to determine these factors by in-situ experiments. We try to measure these local factors; the local temperature is measured by the change of electron transmissivity due to the thermal diffuse scattering (Takaoka et al., 1990), the local stress is roughly estimated from the local strain of crystal plane measured by the width between the bend contours, and the local current density is determined from the applied current.

\section{Observation in Al-lines.}

3.1 HISTOGRAMS OF GRAIN ORIENTATION - Individual orientation of grains in Al-lines can be measured by selected area diffraction with the ultra-HVEM, even if the lines are covered with a thin amorphous layer. The orientation of grains can be also measured by X-ray diffraction and by surface channeling effect with ions or electrons. In the former case, however, we get information only on the averaged orientation of grains in a wide area, and the latter method cannot be applied if the amorphous layer is covered.

Figure 1 shows how the orientation of Al-grains depends on the manufacturing process of LSI devices. Four samples A, B, C and D were picked up after the following process (Ura et al., 1992). At first, $\mathrm{Al}-\mathrm{Si}-\mathrm{Cu}$ alloy was sputter-deposited by $0.4 \mu \mathrm{m}$ on thermally oxidized $\mathrm{Si}$ wafers. 

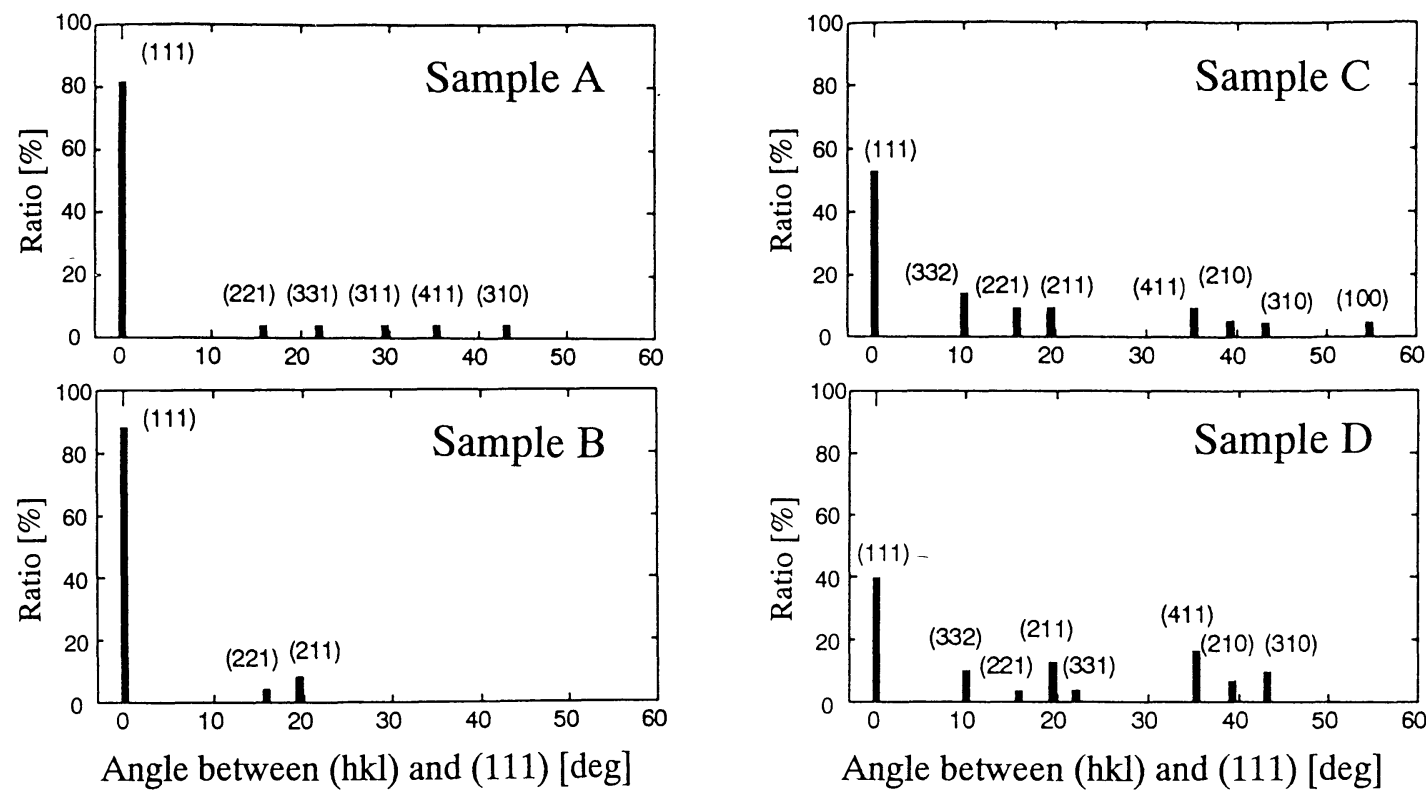

Fig. 1. - Histograms of grain orientation on Al-lines in each step for manufacturing process of an LSI device.

Sample A: after patterning of $\mathrm{Al}$-lines.

Sample B: after sinter at $450{ }^{\circ} \mathrm{C}$ for 15 minutes.

Sample C: after deposition of phospho-silicate-glass (PSG) layer about $0.4 \mu \mathrm{m}$ in thickness by normal-pressure-condition chemical vapor deposition (CVD) at $400{ }^{\circ} \mathrm{C}$.

Sample D: after deposition of SiN layer on the PSG layer about $0.75 \mu \mathrm{m}$ in thickness by plasmaenhanced CVD at $350^{\circ} \mathrm{C}$. For sample $\mathrm{D}$, we prepared another sample whose passivation layer is thinned by plasma etching, because a thick passivation layer blurs the diffraction image considerably even with the ultra-HVEM.

Figure 1 is the histograms for index of grain surface, which is measured and calibrated by the surface index of the Si substrate. The number of measured grains are about 30 in each sample. It is seen that there is a remarkable difference between the histograms of grain orientations before and after deposition of passivation layers. The Al-grains strongly orient in the direction $<111>$ before the deposition of passivation because $\{111\}$ planes have the lowest surface energy in the fcc structure. After the deposition of the passivation layer which induces a temperature rise, it is however seen that this orientation relaxes fairly. This shows that the Al-grains grow and change their orientation during the manufacturing process of LSI devices. From the X-ray diffraction, this difference could not be measured, because the signal level for higher order indexes is less than the level of background.

Next example is the relation between the generation rate of voids by stress-migration and the orientation of grains. It is known that the thick SiN passivation layer made by plasma-CVD exerts a big compressive force to Al-lines the intensity of which being about $200 \mathrm{MPa}$. This force is expected to displace $\mathrm{Al}$-atoms and generate stressmigration voids. We examined the index of grains surrounding voids as well as the index of void surface. The upper histogram in figure 2 shows the distribution of indexes of grains in which voids are not present, while the lower histrogram refers to grains in which voids are generated. In each histogram, the number of measured grains are about 30 . 

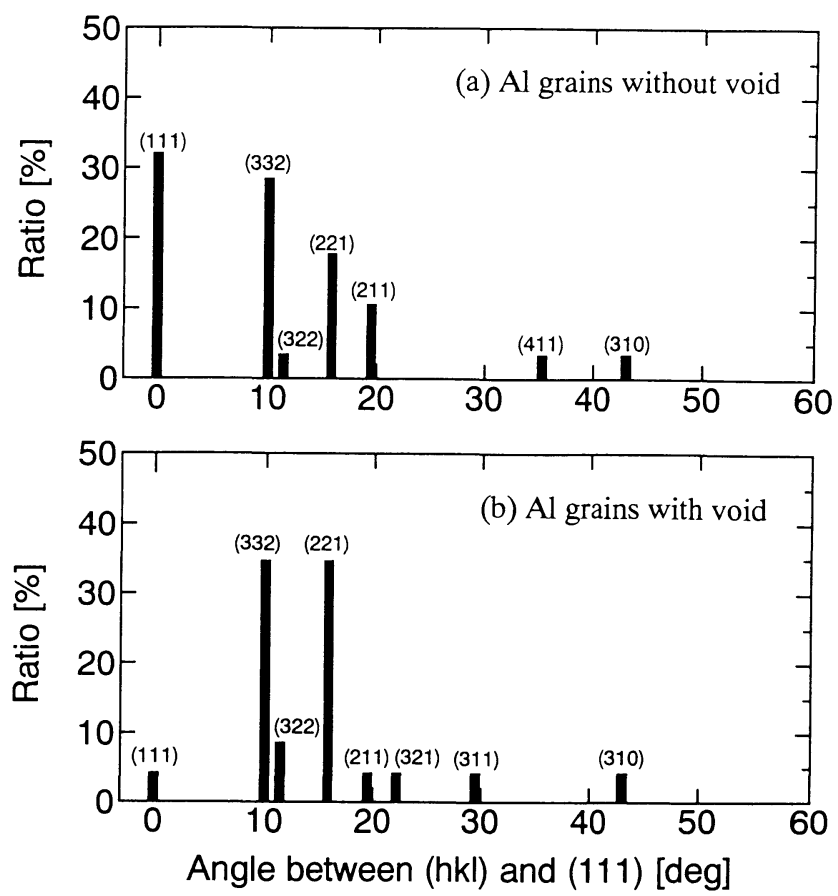

Fig. 2. - Histograms of grain orientation on Al-lines in which voids did not generate (a) and generated (b).

There is a remarkable difference between both histograms; that is, the incidence of voids is low for the grains whose index is $\{111\}$. The voids tend to generate in the grains whose index is high. This result agrees with the empirical fact that the grains oriented near $\{111\}$ are tough for the migrations, and in the manufacturing process, the researchers try to realize highly-oriented lines (Wilson et al., 1991).

3.2 IN-SITU OBSERVATION OF ELECTROMIGRATION - Next example is the in-situ observation of electromigration in Al-lines by ultra-HVEM. We try to observe under such a condition as we cannot measure with usual TEMs, SEMs or optical microscopes; we pay attention to how does the generation of electromigration voids depend on the crystallographic structure in Al-lines under the condition that the Al-lines are covered with the passivation layer.

Figure $3 \mathrm{a}$ and $\mathrm{b}$ shows the dimension of cross section and the top view picture of circuits. The $\mathrm{Al}-1 \% \mathrm{Si}$ lines are covered by the PSG layer of about $0.3 \mu \mathrm{m}$ in thickness. The chip is thinned from the Si-substrate side by chemical etching, then the terminal pads are connected with the external lines to supply the current.

In experiment, we applied the step-stress test; the appleid voltage is increased stepwise by appropriate intervals until the $\mathrm{Al}$-line breaks down. The resistance of the Al-line is monitored and recorded; the resistance also increases stepwise with the current mainly by the temperature rise of Al-line. The fluctuation of resistance is also observed when the voids move, but our data are not accurate enough to discuss the correspondence between the electromigration and the electron radiation effects.

Electromigration is thought to occur preferentially along the grain boundaries because of a lower activation energy. However, when the line is so called bamboo structure, there is no grain 


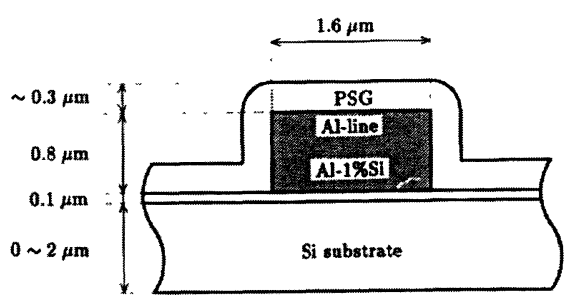

Cross-section of Al-line

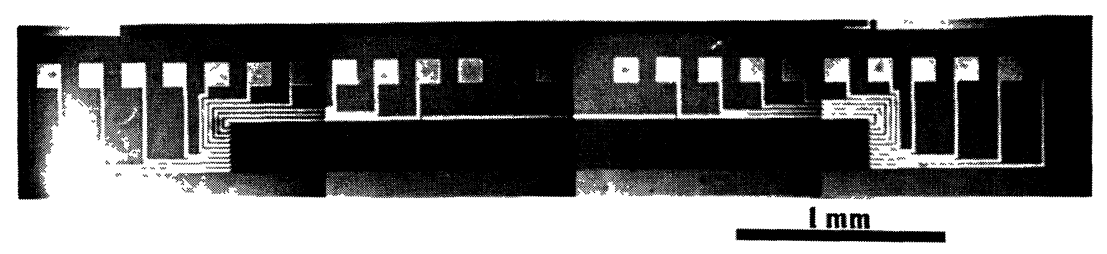

Al-line circuits used in-situ observation of electromigration

Fig. 3. - Cross-section of tested Al-line with passivation layer and the top view of Al-lines by an optical microscope. The current for in-situ experiment of electromigration is supplied from the pads in both sides.

boundary contributing to electromigration. In such cases, the main mechanism of electromigraiton has not been understood yet, especially when the surface is tightened by a passivation layer.

Let us show the typical micrographs of in-situ observation of electromigration. Figure 4 shows the generation of voids and the change of grain shape in an Al-line. When the current density is $4.1 \mathrm{MA} / \mathrm{cm}^{2}, 240 \mathrm{~min}$ after the beginning of the step-stress test, a void appears as expected at the triple junction of grains shown with an arrow in (a). Regardless of the cover by passivation layer, the voids tend to generate in such types of triple junctions where atoms flow in through one boundary and flow out through two ones. Then, some voids generate at the interface between the Al-line and the passivation layer as shown in (b). We suppose that the dominant pass of migration is along the interface in the case of Al-lines with bamboo structure. After the void generation by electromigration, the shape of grains and the position of grain boundary change gradually. This is shown in (c) and (d).

Figure 5 suggests that the migration depends on the tightness of the interface between the line and the passivation. This sample has a small gap at the interface shown with an arrow in (a) before supplying current. When the current density is increased up to about $3.1 \mathrm{MA} / \mathrm{cm}^{2}$, a value of which is considerably less than in the case of figure 4 , the void generates in interface as shown in (b). Furthermore, we can see a slit-like void generated inside the grain. Such a void growth with current flow is shown in (b) - (e).

Figure 6 shows the movement of voids; (a) is the micrograph after $124 \mathrm{~min}$ from the beginning of the step-stress test, where the current density is $4.1 \mathrm{MA} / \mathrm{cm}^{2}$, and $(\mathrm{g})$ is just after the disconnection at $132 \mathrm{~min}$. As the position of the grain boundary is not clear in this micrograph, the boundaries 


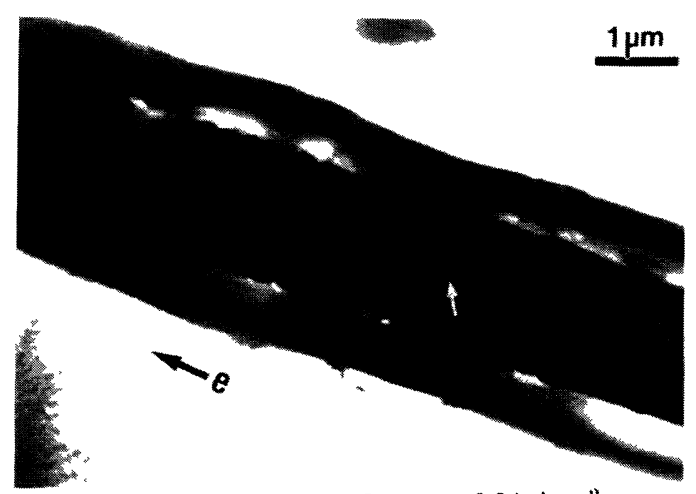

a $\quad \mathrm{t}=240 \mathrm{~min} . \mathrm{J}<4.2 \mathrm{MA} / \mathrm{cm}^{2}$

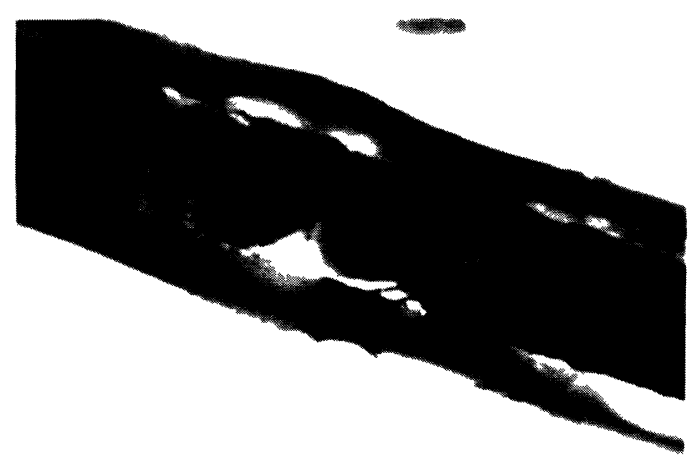

c $\quad t=320 \mathrm{~min} . \mathrm{J}=4.3 \mathrm{MA} / \mathrm{cm}^{2}$

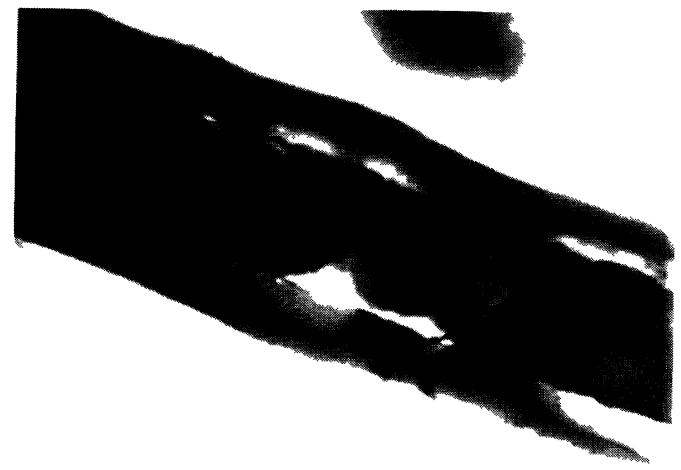

b $\quad t=290 \mathrm{~min} . \mathrm{J}=4.3 \mathrm{MA} / \mathrm{cm}^{2}$

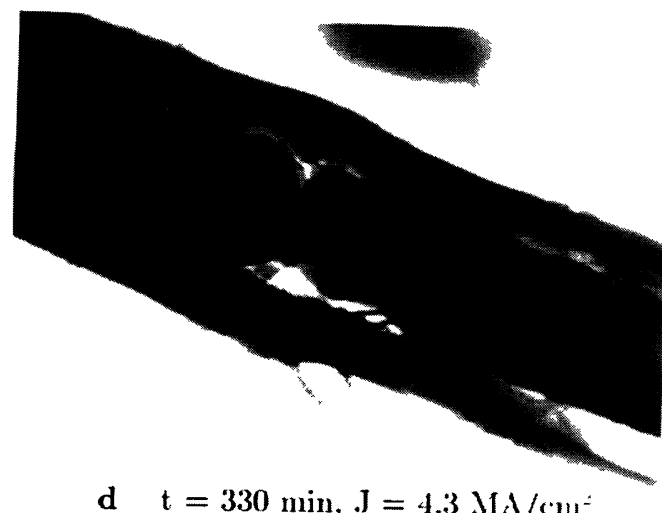

Fig. 4. - In-situ observation of voids in step-stress test to current.

are underlined by broken lines. Furthermore, there exists a grain boundary at the position between two arrows in (a) before the current is applied. The grain marked A is scarcely changed during observation. In (b), a slit-like-void generated in the inside of the grain and then this slit changed into a grain boundary. From (a) to (e), it is seen that voids move to the up-stream for electron flow. This coincides to the fact that $\mathrm{Al}$-atoms migrate to the down-stream due to the momentum of electron collision. Just before the disconnection, the current density concentrates to the narrow position in (f), and the line at that point melted and broke down as shown in (g).

In this experiment, a considerable raise of temperature is estimated from the decrease of intensity and the blur of Al-line image (Takaoka, 1990). The exact local temperature cannot be measured. The grains, however, are in solid state because the Bragg contrast can be observed as shown in the left sides of (e) and (f).

Observed results for the Al-line with bamboo structure are summarized as follows:

1) Voids mainly generate at grain boundaries close to the interface between the passivation layer and the Al-line.

2) Voids move in the direction of the up-stream of electron flow.

3) Atoms mainly migrate through the interface, but sometimes, slit-like voids appear inside grains and move up to the grain boundary.

4) Tightness of interface affects the current density where the migration becomes active. 


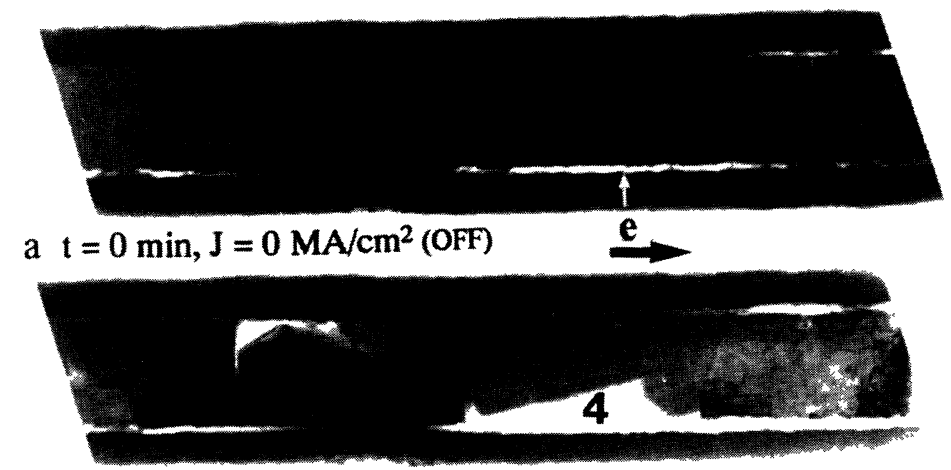

b $\mathrm{t}=10 \mathrm{~min}, \mathrm{~J}=3.1 \mathrm{MA} / \mathrm{cm}^{2}-(\mathrm{OFF})$

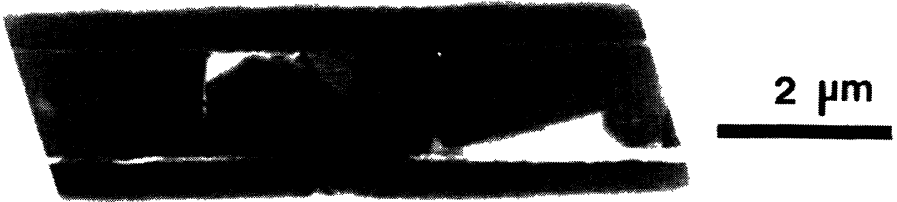

c $\mathrm{t}=110 \mathrm{~min}, \mathrm{~J}=2.0 \mathrm{MA} / \mathrm{cm}^{2}$

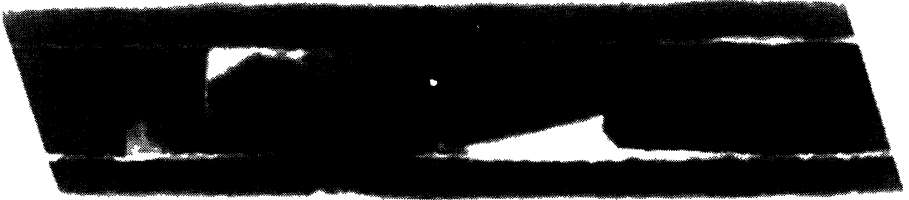

d $\mathrm{t}=210 \mathrm{~min}, \mathrm{~J}=2.7 \mathrm{MA} / \mathrm{cm}^{2}$

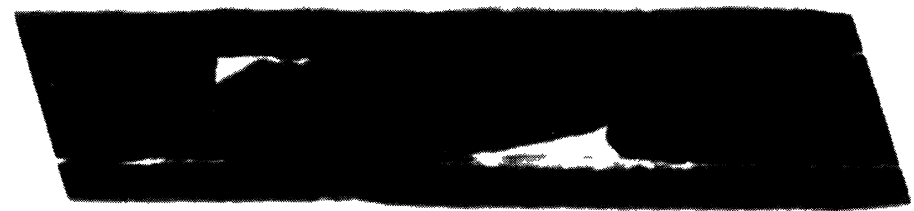

e $\mathrm{t}=220 \mathrm{~min}, \mathrm{~J}=2.9 \mathrm{MA} / \mathrm{cm}^{2}-(\mathrm{OFF})$

Fig. 5. - Generation of voids along the interface between Al-line and passivation layer and growth of slitlike void inside grain.

\section{Conclusion.}

We showed some examples of the perpendicular observation of the process layers of LSI devices, Al-lines under passivation layers. It is seen that the ultra-HVEM is valuable to carry out the investigation of multi-layer devices or to observe Al-lines in-situ under applied stress, current and temperature.

The individual grains in Al-lines change their crystallographic orientations during the manufacturing processes and the orientation parallel to $\{111\}$ in as-deposited Al-layer is released after deposition of passivation layers. From the in-situ observation of electromigration in Al-lines with bamboo structure, we guess that migration occurs mainly along the interface between the Al-line and the passivation layer. Sometimes $\mathrm{Al}$-atoms are however observed to migrate in the bulk, accompanying the movement of slit-like voids in the grain. 


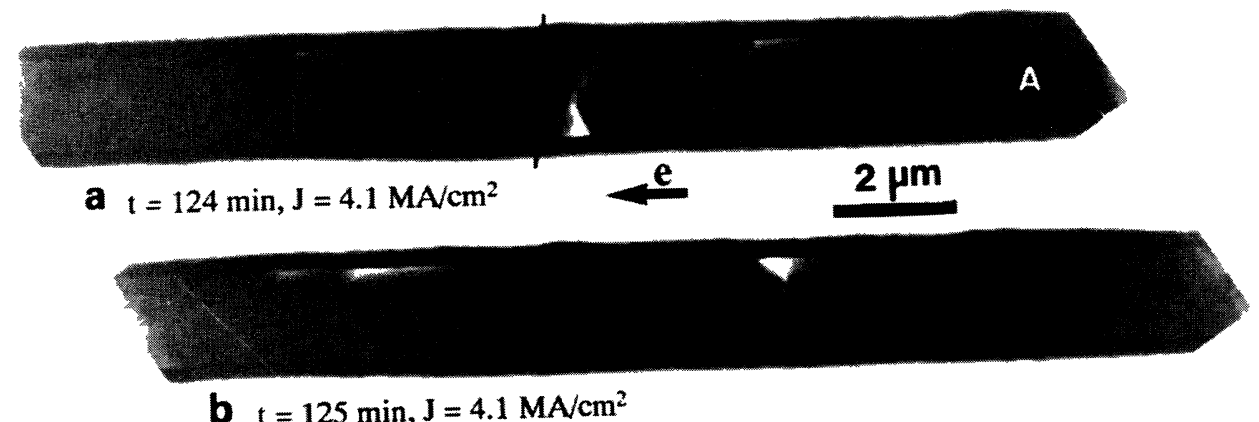

b $\mathrm{t}=125 \mathrm{~min}, \mathrm{~J}=4.1 \mathrm{MA} \mathrm{cm}^{2}$

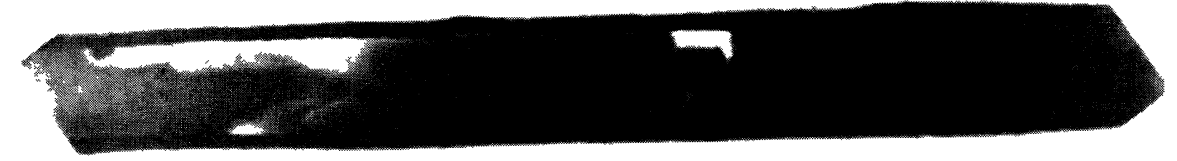

C $\mathrm{t}=126 \mathrm{~min}, \mathrm{~J}=4.1 \mathrm{MA} / \mathrm{cm}^{2}$
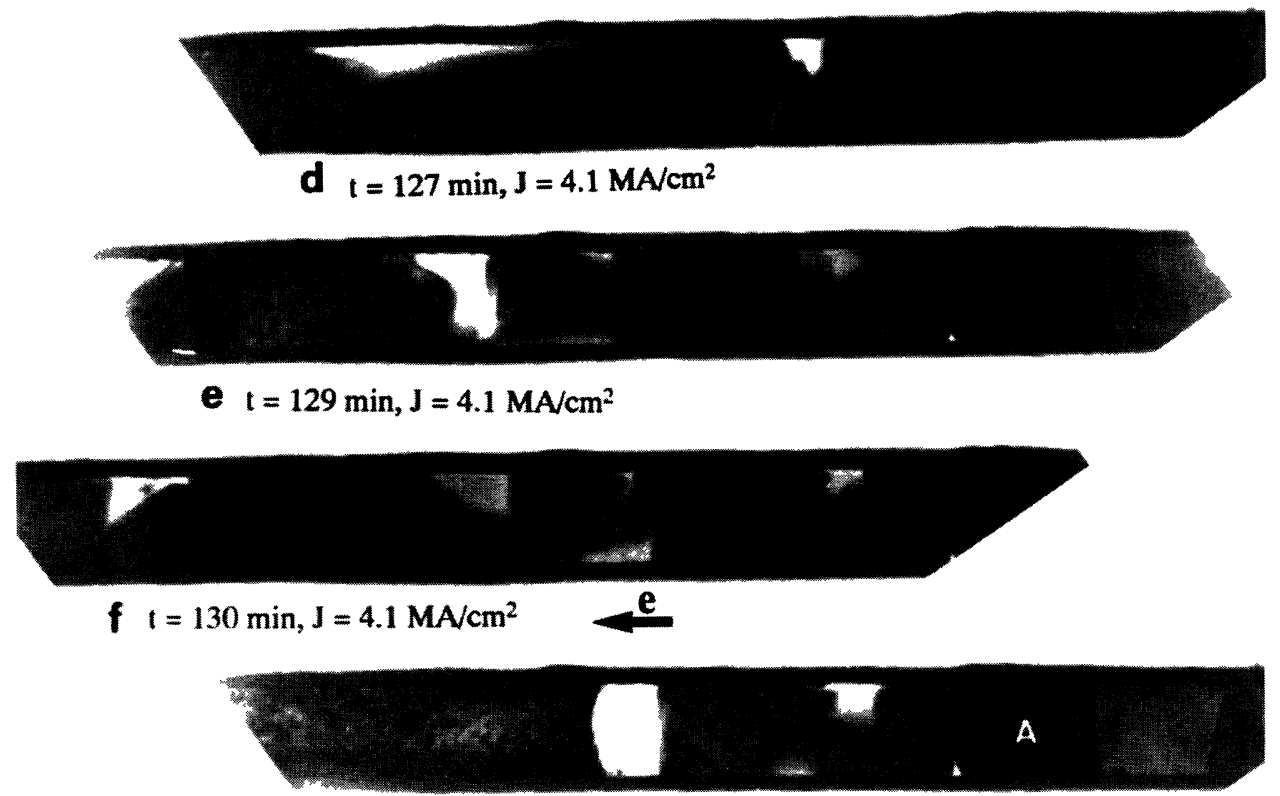

$$
\text { g } \mathrm{t}=132 \min (\text { disconnection), } \mathrm{J}=0
$$

Fig. 6. - Movement of voids by electron flow and disconnection of Al-line.

\section{Acknowledgements.}

The authors would like to thank Dr. H. Egawa of Toshiba Co. (Integrated Circuit Advanced Process Eng. Dept.) for providing the samples of LSI devices and also thank Dr. K. Yoshida of the Research Center of ultra-HVEM in Osaka Univ. for his help in the observation with ultraHVEM. 


\section{References}

[1] Fujita H., Tabata T., Yoshida K., Sumida N. and KatagiRi S., Japan J. appl. Phys. 11 (1972) 1522.

[2] OKABAyashi H., TANiKaWA A., MORI H. and Fujita H., Ultramicroscopy 39 (1991) 306.

[3] REIMER L. and SOMMER K.H., Z. Naturforsch 23a (1968) 1969.

[4] TAKAOKA A. and URA K., J. Electron Microsc. 39 (1990) 69.

[5] TAKAOKA A. and URA K., Ultramicroscopy 39 (1991) 299.

[6] URA K., TAKaOKa A., Yoshida K. and WAdA J., Proc. Int. Conf. Microcircuit Eng. (1992).

[7] WILSON R.V. and WEISS B.L., Vacuum 42 (1991) 719. 